\title{
NEUROTRANSMITTERS IN THE CENTRAL NERVOUS SYSTEM: BASIC KNOWLEDGE REVISITED
}

\author{
Maja Lozić Đurićl \\ ${ }^{1}$ Institute of Pharmacology, Clinical Pharmcology and Toxicology; Faculty of Medicine, University of Belgrade, Serbia \\ Contact e-mail: mlozic@med.bg.ac.rs
}

\section{The chemistry within us}

"The hearts of two frogs were isolated, the first with its nerves, the second without. Both hearts were attached to Straub cannulas filled with a little Ringer solution. The vagus nerve of the first heart was stimulated for a few minutes. Then the Ringer solution that had been in the first heart during the stimulation of the vagus was transferred to the second heart. It slowed and its beats diminished just as if its vagus had been stimulated. Similarly, when the accelerator nerve was stimulated and the Ringer from this period transferred, the second heart speeded up and its beats increased" [1].

This elegant experiment performed by Otto Loewi is considered to be the Rosetta stone of chemical transmission concept and has provided the first evidence that nerves do not influence the heart directly, but liberate specific chemical substances from their terminals. Today, when neurotransmitters are inseparable part of theories of how our brain works, it is difficult to understand that it took more than thirty years of scientific disputes between neurophysiologists and pharmacologists to prove that synaptic transmission is chemical rather than electrical. The great victory of pharmacologists in a war called "the war of sparks and soups" [2,3] happened in 1936, when Otto Loewi and Sir Henry Dale were awarded the Nobel Prize for establishing chemical synaptic transmission.

Given the fact that acetylcholine was the first neurotransmitter to be discovered [4], it is not surprising that the classic criteria for neurotransmitters were based on the properties of this particular molecule.

Table 1. Small-molecule neurotransmitters
Although conventional definition describes a neurotransmitter as a substance that satisfies the following criteria $[5,6]$ :

1. It is synthesized and stored within the presynaptic neuron

2. It is released by nerve stimulation in a calcium-dependent manner

3. It binds to specific receptor(s) localized presinaptically or postsinaptically

4. Its exogenous application mimics the postsynaptic effect of presynaptic stimulation

5. Specific receptor antagonists block the effects of endogenous (synaptically released) or exogenous (externally applied) substance

6. Its action(s) can be terminated in enzyme-mediated way or by the cellular uptake mechanism, the increasing knowledge in the field of neuroscience has been continually modifying the understanding of the term "neurotransmitter". Nowadays, we are faced with at least two questions that may sound like a rather perplexing word game, but still need to be properly addressed: Are only neurotransmitters-neurotransmitters? Are neurotransmitters only neurotransmitters?

\section{Size does matter (?)}

The myriad of chemical messengers is often divided into two large categories based simply on their molecular mass: small-molecule and large-molecule transmitters. But, is it only the size of the molecule that makes the difference between these two groups?

\begin{tabular}{||l|ll||}
\hline \multicolumn{4}{|c|}{ Biogenic amines } & Catecholamines & Acetylcholine \\
\cline { 2 - 3 } & & Dopamine \\
& & Noradrenaline \\
\cline { 2 - 3 } & Indoleamine & Adrenaline \\
\cline { 2 - 3 } Amino acids & Imidazoleamine & Serotonin \\
& & Histamine \\
\hline \multirow{2}{*}{ Purines } & Glutamate \\
& & Aspartate \\
& GABA \\
\hline \hline
\end{tabular}


Small-molecule transmitters (Table 1) fit all the above-mentioned conventional criteria for neurotransmitters-they are presynaptically synthesized, stored in small synaptic vesicles that aggregate at presynaptic location and are released into the synaptic cleft in very high concentration where they predominantly bind to ionotropic receptors. Activity of classical neurotransmitter is limited in both temporal and spatial dimension, due to the existence of the potent machinery that swiftly terminates its action. That practically means that enzymes which degrade neurotransmitters, along with uptake mechanisms, limit the action of small-molecule neurotransmitters to synaptic cleft (spatial specificity) and make their half-life last for merely $5 \mathrm{~ms}$ (temporal specificity) [7].

Large-molecule transmitters are also known as neuropeptides. How do they differ from classical neurotransmitters (Table 2)? Unlike small-molecule transmitters, neuropeptides are synthesized and packaged into large dense-core vesicles in the neuronal body. They can be released at synapses in response to electrical activity and affect neighbouring cells, but the recent evidence shows that exocytosis of large dense-core vesicles seems to be a rather rare event [8]. While classical neurotransmitters represent mediators of "wired", synaptic communication, neuropeptide action is not spatially limited, which means it is not confined solely to synapse. This "wireless", extrasynaptic communication between distant cells or groups of cells in the central nervous system (also known as volume transmission), is mediated by neuropeptides released by different mechanisms from many parts of neuron, including cell bodies, nerve endings and dendrites. In short, neuropeptides possess multifaceted nature-they carry the information which can affect the cells of their origin (taking part in autoregulatory processes), but also demonstrate "classical neurotransmitter-like" and "hormone-like" behavior by affecting adjacent and distant brain cells. Apart from not being spatially restricted, neuropeptide action lacks temporal specificity- they are not rapidly degraded and the half-life of certain neuropeptides in the brain, like oxytocin and vasopressin, can exceed 20 minutes [9].

Probably the most picturesque description of differences between classical small-molecule neurotransmitters and neuropeptides is the one by Ludwig and Leng [8]. They explain neurotransmitters as molecules which pass "whispered secrets" from one cell to another, messages important only at a particular place and at particular time. In contrast, neuropeptides contain messages that are transferred from one cell population to another, messages that last and that could be regarded as "public announcements".

Table 2. Neurotransmitter vs. Neuropeptide

\begin{tabular}{|l|l|}
\hline Neurotransmitter & Neuropeptide \\
\hline synthesized in synaptic nerve end & synthesized in neuronal cell body \\
\hline stored in small synaptic vesicles & stored in large dense-core vesicles \\
\hline synaptic release (spatial specificity) & released from nerve endings, soma, dendrites \\
\hline (mostly)binds to ionotropic receptors & (mostly) binds to metabotropic receptors \\
\hline shorter half-life (ms) (temporal specificity) & longer half-life (min) \\
\hline "whispered secrets" & "public announcements" \\
\hline
\end{tabular}

\section{Fighting the dogma}

"Putative neurotransmitter"is a term used for all the molecules that take part in transferring signals between cells of the nervous system and their targets, but which do not comply to conventional definition of neurotransmitter [5]. What is it that makes putative neurotransmitters so unconventional? These substances, more cautiously named "neural messengers" rather than neurotransmitters, are atypical in chemical nature (since they do not belong to amines, amino acids or peptides), biosynthesis, mechanism of action (do not act at receptor proteins) and cellular localization (not stored in vesicles), but they still "mediate synaptic transmission as faithfully as acetylcholine and noradrenaline" [10].

Gases like nitric oxide (NO), carbon oxide (CO) or hydrogen sulfide (H2S) [11] are not stored in synaptic vesicles, are not released by exocytosis and do not bind to specific postsynaptic (or presynaptic) receptors. Synthesized from arginine, glycine, and cysteine, at physiological levels these so-called "gasotransmitters" activate guanylyl cyclase ( $\mathrm{NO}$ and $\mathrm{CO}$ ) to generate cGMP which elicits a variety of responses (including neurotransmission) via cGMP-dependent protein kinases, or they act through cGMP-independent mechanisms (H2S) involving stimulation of intracellular cAMP signaling 
[12]. Neurons that contain nNOS, enzyme that catalyzes production of NO, have been found in various locations throughout the brain. Although nNOS containing neurons make less than $1 \%$ of cortical cell bodies, it has been shown that these neurons ramify extensively, so that majority of cortical neurons are, in fact, exposed to nNOS nerve terminals [13]. Physiological concentrations of $\mathrm{H} 2 \mathrm{~S}$, on the other hand, are supposed to enhance glutamatergic transmission mediated through NMDA receptors [14].

Endocannabinoids (anandamide and 2-arachidonyl-glycerol) are unsaturated fatty acids that take part in several forms of synaptic regulation. Acting via cannabinoid receptors (CB1 and CB2), they prevent communicaton between postsynaptic target cell and its presynaptic input. Endocannabinoids are retrograde signals that are released from postsynaptic neuron in response to depolarization and elevated concentrations of $\mathrm{Ca} 2+$. Once released, they find their way to presynaptic terminal where they bind to $\mathrm{CB} 1$ receptors and inhibit GABA release, inducing transient reduction of neuronal inhibitory response $[5,10]$.

Zinc is a trace element that satisfies several criteria for a neurotransmitter. It is stored in glutamate-containing synaptic vesicles and after being released upon depolarization, it binds to various receptors. Zinc inhibits NMDA receptors, but there are also indices that it modulates GABA and glycine activity.

One of the major findings in chemical transmission and also one of the biggest strikes to conventional understanding of neurotransmitters is that the neurons are not the only participants in intracellular communication within the brain. Glial cells are known to contain glutamate transporters and express several types of receptors for neurotransmitters, but the surprise came when the astrocytes were proven to synthesize their own, novel putative transmitter called d-serine. $\mathrm{D}$-serine indeed is a neurotransmitter-it is synthetized in astrocytes, it is released due to activation of glial cells, it binds to glycine site of NMDA receptor (more potently than glycine itself !), exogenously applied d-serine fully mimics the activity of endogenous molecule and application of a particular antagonist prevents NMDA-mediated glutamatergic transmission.

\section{Lost in translation}

Despite the evidence that date back from the 1960s, there was a strong belief among neuroscientists that one neuron uses only one transmitter, which lasted until late 1970s. This "one neuron-one neurotransmitter" concept, commonly (and unfortunately) known as Dale's principle, was, in fact, Nobel laureate John Eccles`s inter- pretation of Dale's statement that "a neuron is a metabolic unit and operates at all its synapses by the same chemical transmission mechanisms" [15]. Discovery of storage and release of two or more transmitters from the same terminal, process known as co-transmission, brought to a revision of Dale's principle. This "modern version of Dale's principle" claimed that neurons release the same set of transmitters from all of their axon processes, assuming that the identity of the transmitters expressed by neurons is unchanging. Nevertheless, recent discoveries $[16,17,18]$ demonstrate that electrical activity can respecify neurotransmitter expression during development, but also in mature nervous system. The logical question that arises is what happens with postsynaptic receptors if the identity of presinaptically created neurotransmitter changes. It seems like changes in postsynaptic receptor expression accompany changes in transmitter respecification, thus enabling chemical transmission. The functional consequences of these changes in neurotransmitter identity span from fine-tuning of neural circuits to modifications in behaviour. Although molecular mechanisms lying behind activity-dependent neurotransmitter respecification remain unclear, it is quite possible that the understanding of this phenomenon could lead to better understanding of pathophysiology and treatment of certain neurological and psychiatric disorders.

\section{Are neurotransmitters only neurotransmitters?}

Are neurotransmitters bigger than their name? Previous chapters show that neurotransmitters don't need to act on synapse in order to produce effects, but what is important to mention is that neurotransmitters and their receptors exist before the synapses are even created. A great number of neurotransmitters (ranging from classical to putative) are present during development, even at early embryonic stages before neuronal differentiation is accomplished. They can act as paracrine factors, taking part in morphogenesis and regulating cell movement, migration and proliferation.

\section{Conclusion}

Contemporary understanding of the term "neurotransmitter" has gone the long way from acetylcholine and conventional definition and criteria based on acetylcholine features to a present-day explanations that may somehow seem fluid and ambiguous. Most neuroscientists would agree that, in the widest sense, neurotransmitter is a signaling molecule that regulates the electrochemical state of adjacent cells. From this point of view, 
many putative neurotransmitters like "gasotransmitters" or d-serine would fit the criteria for neurotransmitter status. On the other hand, the fact that neurotransmitters affect much more than "adjacent cells" brings to light the need to rethink the existing criteria.

\section{References}

1. Loewi O. An autobiographical sketch. Perspectives in Biology and Medicine 1960; 4:3-25

2. Valenstein ES. The discovery of chemical neurotransmitters. Brain and Cognition 2002; 49:73-95

3. Cook JS. "Spark" vs. "Soup": A Scoop for Soup. News in Physiological Sciences 1986; 1:206-208

4. Tansey EM. Henry Dale and the discovery of acetylcholine. C R Biol 2006; 329(5-6):419-25

5. Purves D, Augustine GJ, Fitzpatrick D, Katz LC, LaMantia AS, McNamara JO, Williams SM, Editors. Neuroscience. Sunderland (MA): Sinauer Associates; 2001.

6. Evian G, Editor. Integrative Neuroscience: Bringing together biological, psychological and clinical models of the human brain. Harwood Academic Publishers, 2005

7. Ludwig M, Leng G. Dendritic peptide release and peptide-dependent behaviours. Nature Rev Neurosci 2006; 7:126-36

8. Leng G, Ludwig M. Neurotransmitters and peptides: whispered secrets and public announcements. J Physiol 2008; 586:5625-32
9. Mens WB, Witter A, van Wimersma Greidanus TB. Penetration of neurohypophyseal hormones from plasma into cerebrospinal fluid (CSF): half-times of disappearance of these neuropeptides from from CSF. Brain Res 1983; 262:143-9

10. Baraňano DE, Ferris CD, Snyder SH. Atypical neural messengers. Trends in Neurosciences 2001; 24(2):99-106

11. Kimura H. Signaling molecules: hydrogen sulfide and polysulfide. Antioxid Redox Signal 2015; 22(5):362-76

12. Li X, Bazer FW, Gao H, Jobgen W, Johnson GA, Li P, et al. Amino acids and gaseous signaling. Amino acids 2009; 37(1):65-78

13. Bredt DS, Hwang PM, Snyder SH. Localization of nitric oxide synthase indicating a neural role for neuronal nitric oxide. Nature 1990; 347:768-70

14. Snyder SH, Ferris CD. Novel neurotransmitters and their neuropsychiatric relevance. Am J Psychiatry 2000; 157(11):1738-51

15. Dale HH. Pharmacology and nerve endings. Proceedings of the Royal Society of Medicine 1935; 28:319-32

16. Samano C, Cifuentes F, Morales MA. Neurotransmitter segregation: Functional and plastic implications. Progress in Neurobiology 2012; 97:277-87

17. Borodinsky LN, Belgacem YH, Swapna I, Sequerra EB. Dynamic regulation of neurotransmitter specification: Relevance to nervous system homeostasis. Neuropharamcology 2014; 78:75-80

18. Spitzer NC. Activity-dependent neurotransmitter respecification. Nat Rev Neurosci 2012; 13:94-106. 